\title{
Effects of Lifestyle Intervention Performed by Community Pharmacists on Glycemic Control in Patients with Type 2 Diabetes: The Community Pharmacists Assist (Compass) Project, a Pragmatic Cluster Randomized Trial
}

\author{
Hiroshi Okada ${ }^{1,2^{*}}$, Mitsuko Onda ${ }^{3}$, Masaki Shoji ${ }^{3}$, Kazuhiko Kotani ${ }^{4}$, Takeo Nakayama ${ }^{2}$, \\ Yasushi Nakagawa ${ }^{5}$, Naoki Sakane ${ }^{1}$ \\ ${ }^{1}$ Department of Preventive Medicine, Clinical Research Institute for Endocrine and Metabolic Disease, National \\ Hospital Organization Kyoto Medical Center, Kyoto, Japan \\ ${ }^{2}$ Department of Health Informatics, Kyoto University School of Public Health, Kyoto, Japan \\ ${ }^{3}$ Clinical Laboratory of Practical Pharmacy, Osaka University of Pharmaceutical Sciences, Osaka, Japan \\ ${ }^{4}$ Division of Community and Family Medicine, Jichi Medical University, Tochigi, Japan \\ ${ }^{5}$ Polon Company, Kobe, Japan \\ Email: *okada.hiroshi.28z@st.kyoto-u.ac.jp, onda@gly.oups.ac.jp, \\ mary_and_chappy2006@yahoo.co.jp, kazukotani@jichi.ac.jp, \\ nakayama.takeo.4a@kyoto-u.ac.jp, nakagawa@polon-c.com,nsakane@kyotolan.hosp.go.jp
}

Received 21 January 2016; accepted 7 March 2016; published 10 March 2016

Copyright (C) 2016 by authors and Scientific Research Publishing Inc.

This work is licensed under the Creative Commons Attribution International License (CC BY).

http://creativecommons.org/licenses/by/4.0/

c) (i) Open Access

\section{Abstract}

Background: Community pharmacists should be involved in diabetes care, while there has been less evidence about whether a brief lifestyle intervention is effective for diabetes care in community pharmacies. Objectives: To examine the effects of brief lifestyle intervention on glycemic control in patients with type 2 diabetes mellitus (T2D) by using a coaching style, provided by community pharmacists. Methods: A prospective, cluster-randomized, controlled trial was conducted in 50 groups of community pharmacies in Japan. In all, 132 patients with T2D (age, 20 - 75 years, $\geq 8.0 \%$ of hemoglobin A1c (HbA1c)) were assigned to the intervention group $(n=90)$ or the usual care group ( $\mathrm{n}=42$ ). The intervention group (IG) underwent brief lifestyle coaching for self-care of T2D for 6 months. The standard care group (CG) received usual care by pharmacists and was given a

*Corresponding author.

How to cite this paper: Okada, H., et al. (2016) Effects of Lifestyle Intervention Performed by Community Pharmacists on Glycemic Control in Patients with Type 2 Diabetes: The Community Pharmacists Assist (Compass) Project, a Pragmatic Cluster Randomized Trial. Pharmacology \& Pharmacy, 7, 124-132. http://dx.doi.org/10.4236/pp.2016.73016 
general newsletter. The primary outcome was changes in HbA1c levels. Results: After 6 months, the IG had significantly improved HbA1c (IG: $-0.6 \pm 0.9$ vs. CG: $-0.2 \% \pm 0.9 \% ; p=0.021$ using the last observation carried forward analysis). Although the number of drugs reduced from $2.3 \pm 0.8$ to $2.0 \pm 1.2$ in the IG, the number increased from $2.3 \pm 1.1$ to $2.5 \pm 1.1$ in the CG $(-0.2 \pm 0.9$ in IG vs. $0.2 \pm 0.6$ in CG; $p=0.023$ ). Conclusions: The brief lifestyle intervention by community pharmacists improved glycemic control in patients with T2D. Community pharmacists may more positively participate as lifestyle coaches for diabetes care.

\section{Keywords}

Diabetes Care, HbA1c, Drug Adherence, Community Pharmacy, Lifestyle Intervention

\section{Introduction}

Type 2 diabetes mellitus (T2D) is one of the major health issues of the $21^{\text {st }}$ century [1]. The International Diabetes Federation (IDF) has estimated that 382 million people have diabetes mellitus in the world and is assuming that the number of patients will continue to increase. Japan also has 7.2 million people with diabetes, one of the largest numbers in the world [2] [3]. Glycemic control, as evaluated by hemoglobin A1c (HbA1c: a marker reflective of long-term glycemic states), is required in patients with T2D in order to reduce the risk and delay onset of the complications related to T2D and to improve quality of life (QOL) [4] [5]. Kumamoto Study, surveyed Japanese T2D patients, indicated the glycemic threshold to prevent the onset and progression of diabetic microangiopathy; HbA1c (JDS) < 6.5\%, Fasting Blood Glucose $<110 \mathrm{mg} / \mathrm{dl}$, and 2-h postprandial blood glucose concentration $<180 \mathrm{mg} / \mathrm{dl}$ [6]. To ensure the glycemic control, healthcare professionals should cooperate with patients with T2D, specifically, regarding lifestyle modifications.

Pharmacies should be located close to patients with T2D so that they can easily access medication and experience psychological barrier to a lesser extent [7]. Therefore, community pharmacists should be involved in diabetes care [8]. However, there is less evidence regarding diabetes care, and this may have been limited by their work. There have been a few studies on the roles of community pharmacists in glycemic control in the US and Australia [9]-[11]. A pioneering study conducted in the US, but was not a randomized controlled trial (RCT) that showed that HbA1c levels reduced with physical assessments and counseling by pharmacists [9]. Another study conducted in the US, which was also not an RCT, showed reduced HbA1c levels with physical assessments by pharmacists [10]. The study conducted in Australia was an RCT and showed a reduction in HbA1c levels in the intervention group with medication review and drug adherence support when compared with the control group [11].

Although many studies have assessed interventions in Western countries, few studies from Eastern countries have been conducted. This is the first RCT on community pharmacies in Japan.

Evidence-based data, for instance, using an RCT design, are necessary in this field. Importantly, the intervention methods used in these studies [9]-[11] are complex and time-consuming. Because of the free-access system in the society, many people use pharmacies; therefore, brief and effective methods are required for lifestyle interventions performed by community pharmacists. For this purpose, lifestyle coaching (giving advice using brochures and continuous support with every visit) can be briefly used. The current study, thus, aimed to examine the effects of brief lifestyle intervention, by community pharmacists on glycemic control, in patients with T2D.

\section{Methods}

\subsection{Study Design}

This study was an unmasked, cluster RCT. The randomized, parallel group trial was performed at 50 community pharmacies in Japan. Diabetic subjects were randomly assigned to either the lifestyle intervention group (IG) or the standard care group (CG). The subjects were followed for 6 months. The randomization was stratified using matched cluster size. The cluster was a community pharmacy that included patients with diabetes every day 
because of the need for prescription drugs. Community pharmacies were listed by three chain pharmacies and we allocated 50 pharmacies to the IG and CG. These community pharmacies were located in fifteen prefectures in Japan. Pharmacists in the attending pharmacy recruited 1 - 7 patients with diabetes by using a leaflet and poster when they visited the pharmacy.

\subsection{Participants}

\subsubsection{Pharmacy Eligibility Criteria}

1) More than 20 patients with T2D visited the pharmacy in 1 month.

2) Credited pharmacists to deliver the training program according to the protocol and Treatment Guide for Diabetes in Japan.

\subsubsection{Patient Eligibility Criteria}

1) Patients with type 2 diabetes visiting pharmacies for their medication for over 6 months.

2) Patients aged 20 - 75 years.

3) Patients with HbA1c level sustained by 8 or more for over 3 months.

\subsubsection{Patient Eligibility Criteria}

1) Serious complications including dialysis.

2) Dementia and mental illness.

\subsection{Setting and Locations where the Data Were Collected}

The recruitment started in March 2011 and August 2011. The intervention for participants started in April 2011, and the last case was completed in December 2012. Patients were recruited by pharmacists by using standardized brochures and posters. Each pharmacy was asked to recruit 6 patients. They provided consent forms to conduct this study among patients. Eligibility was verified by requesting the most recent clinical data measured in a hospital or clinic within the previous 3 months for HbA1c by using a diabetic note.

\subsection{Training}

The pharmacist who attended this study had been trained for 6 hours on a brief version of motivational interviewing (MI) skills in community pharmacies. This training program was a modified version of the MI (Table 1). This program of mostly than 3 minutes was developed for community pharmacies. This program helped the pharmacists' skill and confidence that was useful for advice-giving. Generally speaking, readiness, importance, and confidence are the key factors to change behavior [12]. Our program focused on the improvement of patients' confidence because we thought that all patients in this study had already been getting ready mentally and felt deeply the importance of changing their lifestyle. To improve patients' confidence, pharmacists were trained on how to communicate with patients to give approval for the measures they have already taken in their daily life. This short version of MI skills based on empowerment includes three steps: 1) Using an open question; 2) Setting goals with patients 3 ) Closing with encouraging. This training program included three cases on snacks,

Table 1. Training program for pharmacists.

\begin{tabular}{ccc}
\hline Guidance & Contents & Min. \\
\hline How to educate about healthy Lifestyle & What is a COMPASS Project & 15 \\
Meal (Case 1) & & 90 \\
Exercise (Case 2) & Strategy for fasting between meals & 90 \\
Drinking (Case 3) & Motivation for increasing physical activity & 90 \\
Reflection & Methods for cutting down on alcohol consumption & 15 \\
Total & 300 \\
\hline
\end{tabular}


exercise, and drinking. These cases were discussed in small groups with role-playing, and were completed within 3 minutes. We prepared fourteen types of brochures that were passed to the patients in the IG at every visit.

\subsection{Control Group}

Patients in the CG were given basic explanation on medication and their HbA1c levels were checked at every visit. The CG was sent newsletters with general information, except for that on healthy lifestyle, and just included a simple greeting in order to prevent drop-out from the study.

\subsection{Intervention Group}

Patients in the IG were given pedometers when they attended this study. In addition, their HbA1c levels were checked and were advised about a healthy lifestyle by a pharmacist when they visited the pharmacy for their prescription drugs. Pharmacists who attended this study were given fourteen types of brochures regarding 1) healthy diets and snacks, 2) exercise tips for improving your HbA1c levels, 3) the goal of blood tests, and 4) reducing alcohol consumption. Patients were given one of these and pharmacists provided short explanations on how to use these leaflets. During every patents visit to the pharmacy, the pharmacists motivated their patients to improve their confidence for making change to their lifestyle within a three minute consultation.

OMRON HJ-205IT was the pedometer used. The IG was sent newsletters about healthy lifestyles including diet and physical activity every month during this study period.

In the first visit, a pharmacist in the IG and a patient discussed goal setting and the way of life style changes. Pharmacists provided information using a brochure and the patient decided the method. At the second visit and after every month, pharmacists checked patient's HbA1c levels, whether patients had achieved their goals, and patients' barrier to life style changes. In addition, they discussed new goal setting at every visit.

\subsection{Study Sample}

According to previous intervention studies in community pharmacies that used self-monitoring of blood glucose (SMBG), there was $0.5 \%$ reduction in HbA1c levels in T2D patients [13] [14]. Therefore, it was assumed that there was a $0.5 \%$ reduction in HbA1c levels in the intervention group because of the non-use of SMBG in the study. The present study was designed to detect a HbA1c $0.5 \%$ reduction in participants due to the intervention. Thus, the sample size required was a cluster of 90 with an average of 2 patients with a type 1 error of 5\%, and a $90 \%$ power (beta $=10 \%$ ) at the two-tailed 5\% significance level, that allowed for a withdrawal rate of $10 \%$.

\subsection{Outcome Measure}

The main outcome was change in HbA1c levels. The other outcomes were medication change, medication adherence, life style change, and change in the attitude and knowledge about diabetes. To analyze the effects of the intervention on changes as well as body mass index (BMI), IPAQ [15], Medication adherence: Modified Morisky Scale 5-point response options [16] and DTSQ [17], multilevel mixed effects regression models using Stata and mixed and meqrlogit command were used, after adjusting for covariates.

\subsection{Measurements}

Medication data was collected from the prescription data. HbA1c data were checked on diabetic notes are usually used in Japanese hospitals and clinics for diabetics. These diabetic notes have not only physical and blood test data including HbA1c but also the stage of nephropathy and retinopathy. Diabetics, physicians and pharmacists can determine patients' medical data from these notes. Height and weight were also checked it. BMI was calculated as weight $(\mathrm{kg}) /$ height $^{2}\left(\mathrm{~m}^{2}\right)$.

\subsection{Procedure}

In chain-pharmacies, an explanatory meeting was undertaken for this study after pharmacists agreed to participate. In addition, we made a list of over 90 prospective pharmacies. After allocation, the pharmacists of the IG were trained by the pharmacy MI program for 6 hours in a single day. After this program, the pharmacists in both groups recruited participants and collected informed consent data. When patients agreed, they send the 
coded data on the application form by fax. Each time the patients visited, the pharmacists sent data the same way for a total of 6 months. Patients filled out the guest questionnaire at the beginning and after 6 months.

\subsection{Data Analyses}

All data are presented as mean \pm SD. Primary analyses used the last observation carried forward (LOCF) for missing data. Comparisons between the groups were made with a two-tailed unpaired t-test. A two-tailed paired t-test was used to analyze the difference within groups between the baseline and the 6-month result. A p value less than 0.05 was considered statistically significant. Descriptive statistics (means, standard deviations, and percentages) were included as crude values.

Internal consistency reliability scores were assessed for diabetic knowledge using Cronbach's alpha, where $>0.7$ was considered as minimum and $>0.9$ was desirable. All data analyses were conducted with STATA V.13. We took into account the clustering effect.

\subsection{Randomization}

Simple randomization was performed through the Center of Randomization at the Division of Prevention Medicine of Clinical Research Institute, National Hospital Organization Kyoto Medical Center in Japan.

\subsection{Blinding}

The study could not be blinded for participants and community pharmacists and health providers because of the nature of the intervention. The study team was not involved in the assessment of eligibility. The collected data was blindly analyzed by an independent statistician.

\section{Results}

\subsection{Recruitment and Attribution}

The IG had 26 pharmacies and the CG included 24 pharmacies. Pharmacies in the IG recruited 106 patients, of which 90 patients completed the study. Pharmacies in the CG recruited 57 patients, of which 42 patients completed the study. Figure 1 shows a flow diagram for this study. Table 2 shows Baseline characteristics of individual and cluster level.

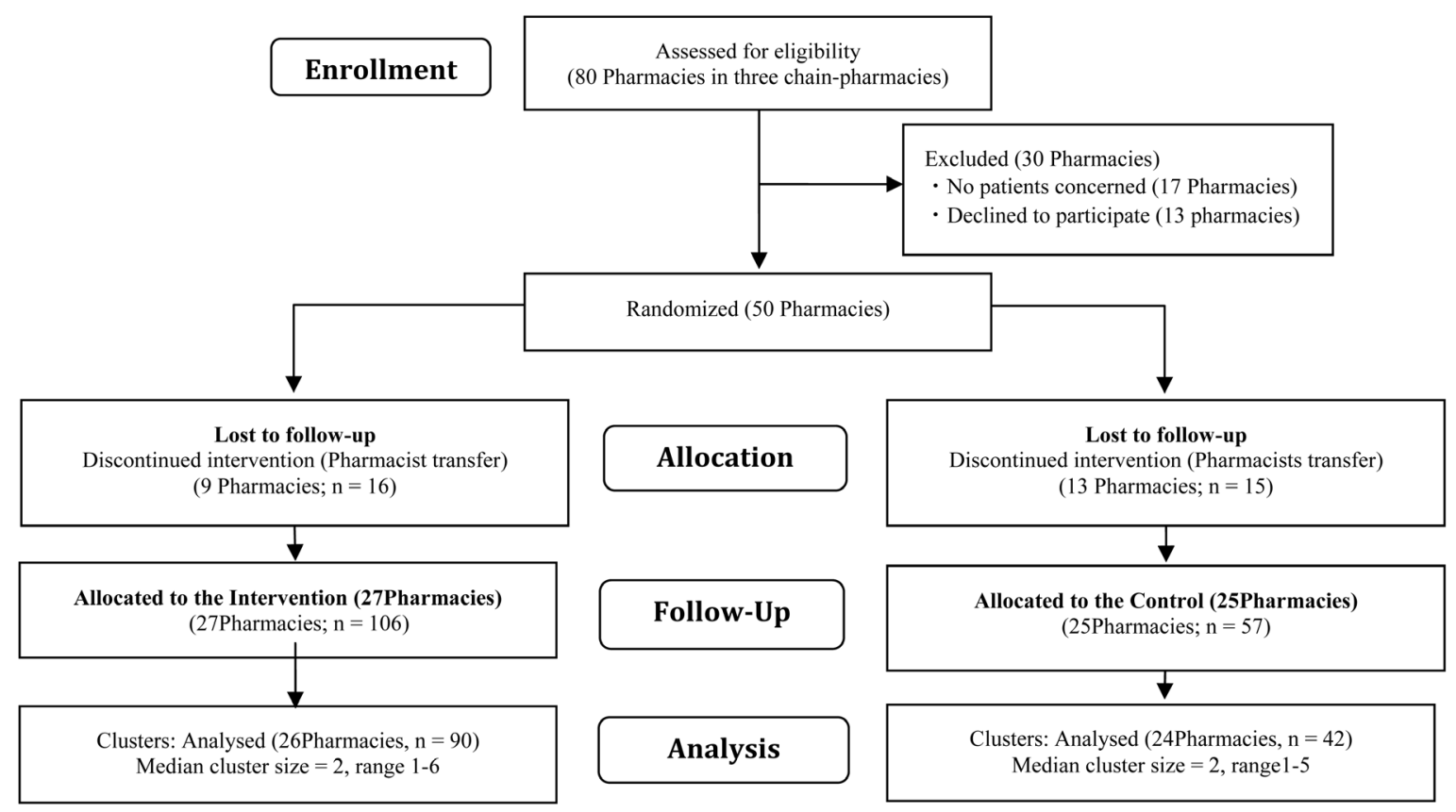

Figure 1. CONSORT flow diagram of the community pharmacists ASSist (COMPASS) project. 


\subsection{Primary Outcome Measures}

Changes in HbA1c levels in the IG were significantly greater than changes in the CG. In addition, the score of diabetic knowledge and the number of diabetes medications was significant by the intervention. There were no differences in other healthy lifestyle indexes between the two groups (Table 3). After adjusted for the clustering

Table 2. Baseline characteristics of individual and cluster level.

\begin{tabular}{|c|c|c|}
\hline Variables & Intervention Group & Control Group \\
\hline Cluster level & $\mathrm{n}=27$ & $\mathrm{n}=25$ \\
\hline \multicolumn{3}{|l|}{ Cluster type $^{\mathrm{a}}(\%)$} \\
\hline Small & $13(48.1)$ & $14(56.0)$ \\
\hline Medium & $7(26.0)$ & $6(24.0)$ \\
\hline Large & $7(26.0)$ & $5(20.0)$ \\
\hline Individual level & $\mathrm{n}=90$ & $\mathrm{n}=42$ \\
\hline Mean (SD) age (years) & $63(9.6)$ & $61(10.8)$ \\
\hline Gender Male/Female & $44 / 46$ & $20 / 22$ \\
\hline \multicolumn{3}{|l|}{ Duration of Diabetes (\%) } \\
\hline-5 years & $21(23.3)$ & 15 (35.7) \\
\hline $6-10$ & $26(28.9)$ & $11(26.2)$ \\
\hline $11-15$ & $21(23.3)$ & $7(16.7)$ \\
\hline $16-20$ & $12(13.3)$ & $6(14.3)$ \\
\hline 21- & $10(11.1)$ & $3(7.1)$ \\
\hline Retinopathy (\%) ${ }^{\mathrm{b}}$ & $18 / 72(25.0)$ & $5 / 35(14.2)$ \\
\hline \multicolumn{3}{|l|}{ Diabetes Medication (\%) ${ }^{\mathrm{C}}$} \\
\hline Insulin & 37/90 (41.1) & $17 / 42(40.5)$ \\
\hline GLP-1 & $0(0)$ & $0(0)$ \\
\hline Sulphonylurea & $46 / 90(51.1)$ & 26/42 (61.9) \\
\hline Biguanide & $37 / 90(41.1)$ & $16 / 42(38.1)$ \\
\hline DPP-4 & 26/90 (28.9) & $14 / 42$ (33.3) \\
\hline Thiazolidinedione & $7 / 90(7.8)$ & $9 / 42(21.4)$ \\
\hline$\alpha-\mathrm{GI}$ & $37 / 90(41.1)$ & $9 / 42(21.4)$ \\
\hline
\end{tabular}

Missing data was not included in the analysis. ${ }^{\mathrm{a}}$ Pharmacies was classified by the number of patients a day. Small: 0 - 50 , Middle: 51 - 100 , Large: 101-. ' Retinopathy was checked through patients' self-report. 'We counted how many kinds of diabetes medication were being taken by each person.

Table 3. Clinical and humanistic parameters of patients at the baseline and the completion of the study.

\begin{tabular}{|c|c|c|c|c|c|c|c|c|c|}
\hline & \multicolumn{2}{|c|}{ Baseline } & \multicolumn{2}{|c|}{ After 6 month } & \multicolumn{2}{|c|}{ Change } & \multicolumn{2}{|c|}{ Difference in change } & \multirow[b]{2}{*}{ P Value } \\
\hline & $\begin{array}{c}\text { IG } \\
(\mathrm{n}=90) \\
\text { Mean (SD) }\end{array}$ & $\begin{array}{c}\text { CG } \\
(\mathrm{n}=42) \\
\text { Mean (SD) }\end{array}$ & $\begin{array}{c}\text { IG } \\
(\mathrm{n}=90) \\
\text { Mean (SD) }\end{array}$ & $\begin{array}{c}\text { CG } \\
(\mathrm{n}=42) \\
\text { Mean (SD) }\end{array}$ & $\begin{array}{c}\text { IG } \\
\text { Mean (SD) }\end{array}$ & $\begin{array}{c}\text { CG } \\
\text { Mean (SD) }\end{array}$ & $\begin{array}{c}\text { Adjusted } \\
\mathrm{B}^{*}\end{array}$ & $95 \%$ CI & \\
\hline $\mathrm{HbA1} c^{\mathrm{a}}$ & $8.7(0.6)$ & $8.7(0.6)$ & $8.0(1.1)$ & $8.4(1.1)$ & $-0.7(0.9)$ & $-0.3(0.9)$ & -0.40 & -0.74 to -0.06 & 0.021 \\
\hline $\mathrm{BMI}^{\mathrm{b}}$ & $24.9(4.9)$ & $24.9(5.8)$ & $25.0(4.7)$ & $24.9(5.7)$ & $0.1(2.0)$ & $0.1(0.5)$ & -0.03 & -0.46 to 0.74 & 0.623 \\
\hline $\mathrm{IPAQ}^{\mathrm{c}}$ & $31.9(32.2)$ & 46.9 (73.8) & $37.8(48.0)$ & 33.5 (35.3) & $5.9(26.2)$ & $-13.4(59.9)$ & 12.3 & -13.4 to 38.1 & 0.348 \\
\hline $\begin{array}{l}\text { Medication } \\
\text { adherence }^{\mathrm{d}}\end{array}$ & $3.5(1.0)$ & $3.5(1.0)$ & $3.7(0.8)$ & $3.3(1.2)$ & $0.1(0.7)$ & $-0.2(0.9)$ & 0.25 & -0.11 to 0.61 & 0.175 \\
\hline DTSQ $^{e}$ & 10.7 (2.9) & $10.8(2.5)$ & $10.1(1.9)$ & $10.0(3.2)$ & $-0.6(3.8)$ & $-0.8(3.6)$ & -0.20 & -1.09 to 1.01 & 0.941 \\
\hline $\begin{array}{l}\text { Medication } \\
\text { Type of drugs }\end{array}$ & $2.3(0.8)$ & $2.3(1.1)$ & $2.0(1.2)$ & $2.5(1.1)$ & $-0.2(0.9)$ & $0.2(0.6)$ & -0.40 & -0.8 to -0.1 & 0.023 \\
\hline Knowledge $^{\mathrm{g}}$ & $3.9(2.1)$ & $3.6(2.3)$ & $5.0(2.4)$ & $3.4(2.0)$ & $1.0(1.9)$ & $-0.3(2.0)$ & 1.4 & 0.4 to 2.4 & 0.005 \\
\hline $\begin{array}{l}\text { Healthy } \\
\text { lifestyle } \\
\text { (7 habits) }^{\mathrm{h}}\end{array}$ & $4.5(1.1)$ & $4.3(1.4)$ & $5.1(1.1)$ & $4.4(1.3)$ & $0.6(1.4)$ & $0.1(2.1)$ & 0.3 & -0.2 to 0.7 & 0.218 \\
\hline
\end{tabular}

${ }^{\mathrm{a}} \mathrm{HbA1c}$, glycated hemoglobin. ${ }^{\mathrm{b}}$ BMI, Body mass index. ${ }^{\mathrm{c}}$ IPAQ, the International Physical Activity Questionnaire. ${ }^{\mathrm{d}}$ Medication adherence, a modified, 6-item. Morisky Medication Adherence Scale (MMAS). ${ }^{\mathrm{e} D T S Q}$, Diabetes Treatment Satisfaction Questionnaire. ${ }^{\mathrm{f}}$ Medication type of drugs, Oral hypoglycemic agent(OHA) was classified into 6 groups. Sulfonylurea (SU), Biguanide (BG), Dipeptidyl Peptidase-4 Inhibitor (DPP-4I), $\alpha$-glucosidase Inhibitor (a-GI), Thiazolidinediones (TZ), and Glinide. ${ }^{\mathrm{g}}$ Knowledge, measured by 10 true or false questions. ${ }^{\mathrm{h}}$ Healthy lifestyle, Lester Breslow: The “Seven Healthy Habits” 1) Get a good night's sleep of seven or eight hours. 2) Exercise 30 minutes at a time, several times a week. 3) Eat moderately to maintain weight in relation to height. 4) Eat breakfast every day. 5) Eat regularly, whether that's two meals a day, three or five. 6) Don’t drink at all or drink moderately. 7) Don't smoke. *Adjusted B, Adjusted for the clustering effect. 
effect the IG had significantly improved HbA1c (IG: $-0.6 \% \pm 0.9 \%$ vs. CG: $-0.2 \% \pm 0.9 \%$; $=0.021$ ).

\subsection{Adverse Events}

No adverse events occurred in either group.

\section{Discussion}

The current RCT demonstrated that with a brief intervention based on a lifestyle coaching style, community pharmacists can play a role in improving glycemic control of patients with T2D, as evaluated by HbA1c. Community pharmacists as lifestyle coaches can contribute to better glycemic control in patients with T2D. An RCT from Australia studied the roles of community pharmacists in glycemic control [11]. Previous studies have used complex and time-consuming lifestyle intervention methods compared to our study on community pharmacists who had been trained for two or more days [9]-[11]. Considering the urgent need for treatment of prevalent T2D using an easy intervention method, few evidence-based studies are underway [13]-[18], and it is worth noting RCTs have shown a positive effect on brief lifestyle coaching performed by community pharmacists, on glycemic control in patients with T2D.

In the current study, community pharmacists used the lifestyle coaching style as an intervention method [19]. The community pharmacists trained for the current study were trained for a brief intervention for $<3$ minutes each time, while patients were visiting the pharmacy. The intervention programs used in the previous studies [9]-[11] are difficult to perform in Japanese pharmacies because of the complex and time-consuming methods (with MI or counseling skills). Thus, considering there were no dropouts and because of the feasibility with significant reductions in HbA1c levels, the current study shows a new direction in the application of brief intervention based on lifestyle coaching style for community pharmacists related to diabetes care.

The change of HbA1c was $-0.7 \%$ in the IG in the current study. Although it was not a large reduction in HbA1c from the baseline, the level is similar to that in previous studies that showed $0.5 \%$ reduction of HbA1c in diabetes education and SMBG by pharmacists [19] and $>1 \%$ reduction in HbA1c levels by lifestyle modification such as physical activity [20]. A reduction of $1 \%$ HbA1c led to $35 \%$ decreased peripheral vascular disease risk [21]. Therefore, the levels observed in the current study may be helpful for reducing the risk of complications and worsening QOL in patients with T2D.

Knowledge of diabetes care significantly increased in patients of the IG. In the IG, community pharmacists explained about diabetes care at every visit and the patients again read the brochures at home. A systematic review reported that diabetes education improves knowledge regardless of glycemic control in some intervention studies; however, some studies did not show similar findings [13] [14] [18]. Most intervention studies on pharmacies assessed diabetes knowledge; however, diabetes knowledge does not indicate behavioral changes and the effects of the advice-giving may become apparent.

A significant reduction in the number of drugs was seen in patients of the IG, but not in patients of the CG. This might be associated with an improvement in HbA1c levels, because physicians might prescribe lesser medications if their patients had reduced HbA1c levels. This is also thought to be one of the important parameters related to intervention to reduce potential medical costs.

One strength of our study includes its RCT design. A limitation of our study includes its small sample size and short duration. Some cases from the CG dropped out, and these were mostly cases that were affected by the earthquake in Japan in 2011; nonetheless, although we continued the study, as there was less bias because this was due to a natural disaster. The use of a questionnaire and self-report survey on knowledge, drug adherence, and lifestyle was also a limitation of the study. This study recruited patients with at-baseline HbA1c levels of over $8.0 \%$. This bias may limit the generalizability of our results.

In summary, the current study demonstrated that the brief lifestyle intervention, based on a lifestyle coaching style by community pharmacists in Japan, could improve glycemic control, as evaluated by HbA1c, in patients with T2D. The brief lifestyle intervention by community pharmacists improved glycemic control in patients with T2D. Community pharmacists may positively participate in diabetes care as lifestyle coaches.

\section{Conclusion}

The brief lifestyle intervention by community pharmacists improved glycemic control in patients with T2D. Community pharmacists may more positively participate in diabetes care as lifestyle coaches. 


\section{Acknowledgements}

The authors would like to acknowledge the participation of the following pharmacies and pharmacists in making this study possible: Nihon Chouzai Co., Ltd., QOL Co., Ltd., Saera Pharmacy Co., Ltd. We'd like to thank Katsushi Fukuoka (Nihon Chouzai), Yasuhiro Ishii (QOL), for adjustment schedule and coordinating pharmacies as well as the study participants for their significant contribution to the trial. And appreciation goes to Ms. Denise Epp (Daiichi University of Pharmacy) for checking the draft of this article in English.

\section{Funding}

This work was supported by Policy-based medical service foundation (Grant number: H23-No. 9).

\section{Conflict of Interests}

The authors have no conflict of interest directly relevant to the content of this article.

\section{Ethics Approval}

Ethics approval was provided by the Ethics Committee of the Kyoto medical center (09-33).

\section{References}

[1] Chen, L., Magliano, D.J. and Zimmet, P.Z. (2011) The Worldwide Epidemiology of Type 2 Diabetes Mellitus-Present and Future Perspectives. Nature Reviews Endocrinology, 8, 228-236. http://dx.doi.org/10.1038/nrendo.2011.183

[2] International Diabetes Federation: IDF Diabetes Atlas Sixth Edition 2013. https://www.idf.org/sites/default/files/EN_6E_Atlas_Full_0.pdf

[3] National Institute of Health and Nutrition, Section of the National Health and Nutrition Survey. http://www0.nih.go.jp/eiken/english/research/project_nhns.html

[4] UK Prospective Diabetes Study (UKPDS) Group (1998) Intensive Blood-Glucose Control with Sulphonylureas or Insulin Compared with Conventional Treatment and Risk of Complications in Patients with Type 2 Diabetes (UKPDS 33). Lancet, 12, 837-853.

[5] Wake, N., Hisashige, A., Katayama, T., Kishikawa, H., Ohkubo, Y., Sakai, M., Araki, E. and Shichiri, M. (2000) CostEffectiveness of Intensive Insulin Therapy for Type 2 Diabetes: A 10-Year Follow-Up of the Kumamoto Study. Diabetes Research and Clinical Practice, 48, 201-210. http://dx.doi.org/10.1016/S0168-8227(00)00122-4

[6] Ohkubo, Y., Kishikawa, H., Araki, E., Miyata, T., Isami, S., Motoyoshi, S., Kojima, Y., Furuyoshi, N. and Shichiri, M. (1995) Intensive Insulin Therapy Prevents the Progression of Diabetic Microvascular Complications in Japanese Patients with Non-Insulin-Dependent Diabetes Mellitus: A Randomized Prospective 6-Year Study. Diabetes Research and Clinical Practice, 28, 103-117. http://dx.doi.org/10.1016/0168-8227(95)01064-K

[7] Eades, C.E., Ferguson, J.S. and O’Carroll, R.E. (2011) Public Health in Community Pharmacy: A Systematic Review of Pharmacist and Consumer Views. BMC Public Health, 21, 582.

[8] Twigg, M.J., Poland, F., Bhattacharya, D., Desborough, J.A. and Wright, D.J. (2013) The Current and Future Roles of Community Pharmacists: Views and Experiences of Patients with Type 2 Diabetes. Research in Social Administrative Pharmacy, 9, 777-789. http://dx.doi.org/10.1016/j.sapharm.2012.10.004

[9] Cranor, C.W., Bunting, B.A. and Christensen, D.B. (2003) The Asheville Project: Long-Term Clinical and Economic Outcomes of a Community Pharmacy Diabetes Care Program. Journal of the American Pharmaceutical Association, 43, 173-184. http://dx.doi.org/10.1331/108658003321480713

[10] Fera, T., Bluml, B.M. and Ellis, W.M. (2009) Diabetes Ten City Challenge: Final Economic and Clinical Results. Journal of the American Pharmaceutical Association, 49, 383-391. http://dx.doi.org/10.1331/JAPhA.2009.09015

[11] Krass, I., Armour, C.L., Mitchell, B., Brillant, M., Dienaar, R. and Hughest, J. (2007) The Pharmacy Diabetes Care Program: Assessment of a Community Pharmacy Diabetes Service Model in Australia. Diabetic Medicine, 24, 677-683. http://dx.doi.org/10.1111/j.1464-5491.2007.02143.x

[12] Rollnick, S., Mason, P. and Butler, C. (1999) Health Behavior Change. Harcourt Health Science, London.

[13] Machado, M., Bajcar, J., Guzzo, G.C. and Einarson, T.R. (2007) Sensitivity of Patient Outcomes to Pharmacist Interventions. Part I: Systematic Review and Meta-Analysis in Diabetes Management. Annals of Pharmacotherapy, 41, 1569-1582. http://dx.doi.org/10.1345/aph.1K151 
[14] Machado, M., Bajcar, J., Guzzo, G.C. and Einarson, T.R. (2007) Sensitivity of Patient Outcomes to Pharmacist Interventions. Part II: Systematic Review and Meta-Analysis in Hypertension Management. Annals of Pharmacotherapy, 41, 1770-1781. http://dx.doi.org/10.1345/aph.1K311

[15] Guidelines for Data Processing and Analysis of the International Physical Activity Questionnaire (IPAQ). http://www.academia.edu/5346814/Guidelines_for_Data_Processing_and_Analysis_of_the_International_Physical_Ac tivity_Questionnaire_IPAQ_Short_and_Long_Forms_Contents

[16] Morisky, D.E., Green, L.W. and Levine, D.M. (1986) Concurrent and Predictive Validity of a Self-Reported Measure of Medication Adherence. MedCare, 24, 67-74. http://dx.doi.org/10.1097/00005650-198601000-00007

[17] Ishii, H., Bradley, C., Riazi, A. and Yamamoto, T. (2000) The Japanese Version of the Diabetes Treatment Satisfaction Questionnaire (DTSQ): Translation and Clinical Evaluation. Journal of Clinical and Experimental Medicine, 192, 809814.

[18] O’Donovan, D.O., Byrne, S. and Sahm, L. (2011) The Role of Pharmacists in Control and Management of Type 2 Diabetes Mellitus: A Review of the Literature. Journal of Diabetology. http://www.journalofdiabetology.org/Pages/Releases/FullTexts/FourthIssue/RA-1-JOD-10-023.aspx

[19] American Diabetes Association Standards of Medical Care in Diabetes_-2015, Volume 38, Supplement 1. http://professional.diabetes.org/admin/UserFiles/0\%20-\%20Sean/Documents/January\%20Supplement\%20Combined F inal.pdf

[20] Boulé, N.G., Haddad, E., Kenny, G.P., Wells, G.A. and Sigal, R.J. (2001) Effects of Exercise on Glycemic Control and Body Mass in Type 2 Diabetes Mellitus: A Meta-Analysis of Controlled Clinical Trials. JAMA, 12, 1218-1227. http://dx.doi.org/10.1001/jama.286.10.1218

[21] Stratton, I.M., Adler, A.I., Neil, H.A., Matthews, D.R.., Manley, S.E., Cull, C.A., Hadden, D., Turner, R.C. and Holman, R.R. (2000) Association of Glycaemia with Macrovascular and Microvascular Complications of Type 2 Diabetes (UKPDS 35): Prospective Observational Study. BMJ, 12, 405-412. http://dx.doi.org/10.1136/bmj.321.7258.405 